\title{
AGGRESSIVE TOTAL PARENTERAL NUTRITION IS ASSOCIATED WITH AN INCREASED INCIDENCE OF NEPHROCALCINOSIS IN VERY LOW BIRTH WEIGHT INFANTS
}

\author{
E.A. Park, H.M. Ahn, S.J. Cho \\ Pediatric Department, Ewha Womans University, Seoul, Republic of Korea
}

Background and aims: As aggressive total parenteral nutrition (TPN) is replaced as the standard protocol, the aim of our study was to determine the effect of aggressive TPN focusing on the immature kidney. We compared the incidence and natural course of nephrocalcinosis (NC) in premature infants from two different nutrition strategies.

Methods: We studied all preterm neonates with a birth weight below $1500 \mathrm{~g}$ who were born from Jan 2006 to Dec 2010 retropectively. Sequential abdominal ultrasonography was performed in all patients. The clinical parameters, maternal factors, respiratory support and duration of furosemide were analyzed. The change of nutrition strategy was also compared with the incidence of NC.

Results: NC was detected in 35 of 101 VLBW infants (34.7\%). Low birth weight was significantly associated with $\mathrm{NC}(\mathrm{P}<0.05)$. The duration of oxygen use, furosemide treatment and TPN were also strongly associated with NC. Concerning the change in our hospital's nutritional strategy since 2008, such as the increase of protein intake in the first week, the incidence of NC was significantly increased after 2008 $(19.5 \%$ vs. $43.3 \%, p<0.05)$. NC persisted in four infants $(4 / 35,11.4 \%)$ after 12 months follow-up period and one infant developed urolithiasis. Among these four infants, three infants received aggressive TPN.

Conclusions: Though NC resolves spontaneously in most patients, persistent progressive renal injury can develop, especially after aggressive TPN. Therefore, early diagnostic evaluation is crucial and close follow up of $\mathrm{NC}$ in preterm neonates are needed. 